Kragujevac Journal of Mathematics

Volume 42(4) (2018), Pages 517-525.

\title{
ZERO-ANNIHILATOR GRAPHS OF COMMUTATIVE RINGS
}

\author{
HOJJAT MOSTAFANASAB ${ }^{1}$
}

\begin{abstract}
Assume that $R$ is a commutative ring with nonzero identity. In this paper, we introduce and investigate zero-annihilator graph of $R$ denoted by $\mathrm{ZA}(R)$. It is the graph whose vertex set is the set of all nonzero nonunit elements of $R$ and two distinct vertices $x$ and $y$ are adjacent whenever $\operatorname{Ann}_{R}(x) \cap \operatorname{Ann}_{R}(y)=\{0\}$.
\end{abstract}

\section{INTRODUCTION}

Throughout this paper all rings are commutative with nonzero identity. In [6], Beck associated to a ring $R$ its zero-divisor graph $G(R)$ whose vertices are the zero-divisors of $R$ (including 0 ), and two distinct vertices $x$ and $y$ are adjacent if $x y=0$. Later, in [3], Anderson and Livingston studied the subgraph $\Gamma(R)$ of $G(R)$ (whose vertices are the nonzero zero-divisors of $R$ ). In the recent years, several researchers have done interesting and enormous works on this field of study. For instance, see $[4,5,9]$. The concept of co-annihilating ideal graph of a ring $R$, denoted by $\mathcal{A}_{R}$ was introduced by Akbari et al. in [1]. As in [1], co-annihilating ideal graph of $R$, denoted by $\mathcal{A}_{R}$, is a graph whose vertex set is the set of all non-zero proper ideals of $R$ and two distinct vertices $I$ and $J$ are adjacent whenever $\operatorname{Ann}_{R}(I) \cap \operatorname{Ann}_{R}(J)=\{0\}$. In the present paper, we introduce zero-annihilator graph of $R$ denoted by $\mathrm{ZA}(R)$. It is the graph whose vertex set is the set of all nonzero nonunit elements of $R$ and two distinct vertices $x$ and $y$ are adjacent whenever $A_{n}(R x+R y)=\operatorname{Ann}_{R}(x) \cap \operatorname{Ann}_{R}(y)=\{0\}$. Note that $\mathrm{ZA}(R)$ is an induced subgraph of $\mathcal{A}_{R}$.

Let $G$ be a simple graph with the vertex set $\mathrm{V}(G)$ and edge set $\mathrm{E}(G)$. For every vertex $v \in \mathrm{V}(G), \mathrm{N}_{G}(v)$ is the set $\{u \in \mathrm{V}(G) \mid u v \in \mathrm{E}(G)\}$. The degree of a vertex $v$ is defined as $\operatorname{deg}_{G}(v)=\left|\mathrm{N}_{G}(v)\right|$. The minimum degree of $G$ is denoted

Key words and phrases. Graphs, commutative rings, zero-annihilator graphs. 2010 Mathematics Subject Classification. Primary: 13A15. Secondary: 16N40.

Received: October 06, 2016.

Accepted: May 22, 2017. 
by $\delta(G)$. Recall that a graph $G$ is connected if there is a path between every two distinct vertices. For distinct vertices $x$ and $y$ of a connected graph $G$, let $\mathrm{d}_{G}(x, y)$ be the length of the shortest path from $x$ to $y$. The diameter of a connected graph $G$ is $\operatorname{diam}(G)=\sup \left\{\mathrm{d}_{G}(x, y) \mid x\right.$ and $y$ are distinct vertices of $\left.G\right\}$. The girth of $G$, denoted by $\operatorname{girth}(G)$, is defined as the length of the shortest cycle in $G$ and $\operatorname{girth}(G)=\infty$ if $G$ contains no cycles. A bipartite graph is a graph all of whose vertices can be partitioned into two parts $U$ and $V$ such that every edge joins a vertex in $U$ to a vertex in $V$. A complete bipartite graph is a bipartite graph with parts $U, V$ such that every vertex in $U$ is adjacent to every vertex in $V$. A graph in which all vertices have degree $k$ is called a $k$-regular graph. A graph in which each pair of distinct vertices is joined by an edge is called a complete graph. Also, if a graph $G$ contains one vertex to which all other vertices are joined and $G$ has no other edges, is called a star graph. A clique in a graph $G$ is a subset of pairwise adjacent vertices and the number of vertices in a maximum clique of $G$, denoted by $\omega(G)$, is called the clique number of $G$. The chromatic number of $G$, denoted by $\chi(G)$, is the minimum number of colors needed to color the vertices of $G$ so that no two adjacent vertices have the same color. Obviously, $\chi(G) \geq \omega(G)$.

\section{Some Properties of ZA $(R)$}

Recall that, an empty graph is a graph with no edges. A Bézout ring is a ring in which all finitely generated ideals are principal.

Theorem 2.1. Let $R$ be a ring. If $\mathrm{ZA}(R)$ is an empty graph, then $R$ is a local ring and $\operatorname{Ann}_{R}(x) \neq\{0\}$ for every nonunit element $x \in R$. The converse is true if $R$ is a Bézout ring.

Proof. Assume that $\mathrm{ZA}(R)$ is empty. Let $\mathfrak{m}_{1}, \mathfrak{m}_{2}$ be two distinct maximal ideals of $R$. Then $\mathfrak{m}_{1}+\mathfrak{m}_{2}=R$ implies that there exist $x \in \mathfrak{m}_{1}$ and $x_{2} \in \mathfrak{m}_{2}$ such that $x+y=1$. So $x$ and $y$ are adjacent, which is a contradiction. Hence $R$ is a local ring. Let $\mathfrak{m}$ be the maximal ideal of $R$ and $x$ be an element of $\mathfrak{m}$. Suppose that $\operatorname{Ann}_{R}(x)=\{0\}$. Then $\left\{x^{n} \mid n \in \mathbb{N}\right\}$ is an infinite clique in $\mathrm{ZA}(R)$ that is a contradiction. So $\operatorname{Ann}_{R}(x) \neq\{0\}$.

Suppose that $R$ is a local Bézout ring and $\operatorname{Ann}_{R}(x) \neq\{0\}$ for every nonunit element $x \in R$. Let $x, y$ be two vertices in $\mathrm{ZA}(R)$. Then $x, y \in \mathfrak{m}$. Hence $R x+R y=R z$ for some nonzero nonunit element $z \in R$. So $x, y$ are not adjacent which shows that $\mathrm{ZA}(R)$ is empty.

Remark 2.1. Suppose that $R$ has a nontrivial idempotent element $e$. Then $e+(1-e)=1$ implies that $e$ and $1-e$ are adjacent. Hence $\operatorname{deg}_{\mathrm{ZA}(R)}(e) \geq 1$ and so $\mathrm{ZA}(R)$ is not an empty graph.

Remark 2.2. Let $R$ be a ring. Notice that if $R$ is an Artinian ring or a Boolean ring, then $\operatorname{dim}(R)=0$. By [2, Theorem 3.4], $\operatorname{dim}(R)=0$ if and only if for every $x \in R$ there exists a positive integer $n$ such that $x^{n+1}$ divides $x^{n}$. Therefore, every nonzero 
nonunit element of a zero-dimensional ring has a nonzero annihilator. Hence, if $R$ is a zero-dimensional chained ring, then $\mathrm{ZA}(R)$ is an empty graph.

Let $\mathrm{Z}^{*}(R)$ denote the zero divisors of $R$ and $\mathrm{Z}(R)=\mathrm{Z}^{*}(R) \cup\{0\}$.

Theorem 2.2. Let $R$ be a ring and $S$ be a multiplicative closed subset of $R$ such that $S \cap \mathrm{Z}(R)=\{0\}$. Then $\mathrm{ZA}(R) \simeq \mathrm{ZA}\left(R_{S}\right)$.

Proof. Define the vertex map $\Phi: \mathrm{V}(\mathrm{ZA}(R)) \rightarrow \mathrm{V}\left(\mathrm{ZA}\left(R_{S}\right)\right)$ by $x \mapsto \frac{x}{1}$. We can easily verify that $x=y$ if and only if $\frac{x}{1}=\frac{y}{1}$. Also, it is easy to see that $\operatorname{Ann}_{R}(x) \cap \operatorname{Ann}_{R}(y)=$ $\{0\}$ if and only if $\operatorname{Ann}_{R_{S}}\left(\frac{x}{1}\right) \cap \operatorname{Ann}_{R_{S}}\left(\frac{y}{1}\right)=\left\{\frac{0}{1}\right\}$.

Theorem 2.3. Let $R$ be a Bézout ring with $|\operatorname{Max}(R)|<\infty$ such that $\delta(\mathrm{ZA}(R))>0$. Then $\mathrm{ZA}(R)$ is a finite graph if and only if every vertex of $\mathrm{ZA}(R)$ has finite degree.

Proof. The "only if" part is evident.

Suppose that each vertex of $\mathrm{ZA}(R)$ has finite degree. If $\operatorname{Ann}_{R}(x)=\{0\}$ for some nonzero nonunit element $x \in R$, then $x$ is adjacent to all vertices of $\mathrm{ZA}(R)$ that implies $\mathrm{ZA}(R)$ is a finite graph. Assume that $\operatorname{Ann}_{R}(x) \neq\{0\}$ for each nonzero nonunit element $x \in R$. We claim that $\operatorname{Jac}(R)=\{0\}$. On the contrary, assume that there exists a nonzero element $a \in \operatorname{Jac}(R)$. Since $\mathrm{ZA}(R)$ has no isolated vertex, $a$ is adjacent to another vertex, say $b$. Since $R$ is a Bézout ring, $R a+R b$ is generated by a nonzero nonunit element $c$ of $R$ and so $\operatorname{Ann}_{R}(R a+R b)=\operatorname{Ann}_{R}(c) \neq\{0\}$, which is impossible. So $\operatorname{Jac}(R)=\{0\}$. Hence by Chinese Remainder Theorem we have $R \simeq F_{1} \times F_{2} \times \cdots \times F_{n}$, where $F_{i}$ 's are fields and $n=|\operatorname{Max}(R)|$. Let $0 \neq u \in F_{1}$. Then $(u, 0, \ldots, 0)$ and $(0,1, \ldots, 1)$ are adjacent. Since $(0,1, \ldots, 1)$ has finite degree, so $F_{1}$ is a finite field. Similarly we can show that $F_{i}$ 's are finite fields. Consequently $R$ has finitely many nonzero nonunit elements and the proof is complete.

Theorem 2.4. Let $R$ be a Bézout ring with $|\operatorname{Max}(R)|<\infty$. Then the following conditions are equivalent:

(a) $\mathrm{ZA}(R)$ is a bipartite graph with $\delta(\mathrm{ZA}(R))>0$;

(b) $\mathrm{ZA}(R)$ is a complete bipartite graph;

(c) $R \simeq F_{1} \times F_{2}$ where $F_{1}$ and $F_{2}$ are two fields.

Proof. (a) $\Rightarrow$ (c) Suppose that $\mathrm{ZA}(R)$ is a bipartite graph with $\delta(\mathrm{ZA}(R))>0$. If $\operatorname{Ann}_{R}(x)=\{0\}$ for some nonzero nonunit element $x$ of $R$, then $\left\{x^{n} \mid n \in \mathbb{N}\right\}$ is an infinite clique that is a contradiction. Then, for every nonzero nonunit element $x$ of $R$ we have $\operatorname{Ann}_{R}(x) \neq\{0\}$. Similar to the proof of Theorem 2.3 we can show that $R=F_{1} \times F_{2} \times \cdots \times F_{n}$, where $F_{i}$ 's are fields and $n=|\operatorname{Max}(R)|$. Clearly $n \neq 1$. If $n \geq 3$, then $\{(0,1, \ldots, 1),(1,0,1, \ldots, 1),(1,1,0,1, \ldots, 1)\}$ is a clique in $\mathrm{ZA}(R)$, a contradiction. So $R \simeq F_{1} \times F_{2}$.

(c) $\Rightarrow$ (b) Suppose that $R \simeq F_{1} \times F_{2}$ where $F_{1}$ and $F_{2}$ are two fields. Every vertex in $\mathrm{ZA}(R)$ is of the form $(u, 0)$ or $(0, v)$ where $0 \neq u \in F_{1}$ and $0 \neq v \in F_{2}$. Also, two vertices $(u, 0)$ and $(0, v)$ are adjacent. On the other hand, every two vertices $\left(u_{1}, 0\right),\left(u_{2}, 0\right)$ cannot be adjacent. 
$(\mathrm{b}) \Rightarrow(\mathrm{a})$ is clear.

Theorem 2.5. Let $R$ be a ring and $n \geq 2$ be a natural number. Then

$$
\operatorname{girth}\left(\mathrm{ZA}\left(M_{n}(R)\right)\right)=3 .
$$

Proof. For $n=2$, the following matrices are pairwise adjacent in $\mathrm{ZA}\left(\mathrm{M}_{2}(R)\right)$ :

$$
\left(\begin{array}{ll}
1 & 0 \\
0 & 0
\end{array}\right),\left(\begin{array}{ll}
0 & 0 \\
1 & 0
\end{array}\right) \text { and }\left(\begin{array}{ll}
0 & 1 \\
0 & 1
\end{array}\right)
$$

For $n \geq 3$, the following matrices are pairwise adjacent in $\mathrm{ZA}\left(\mathrm{M}_{n}(R)\right)$ :

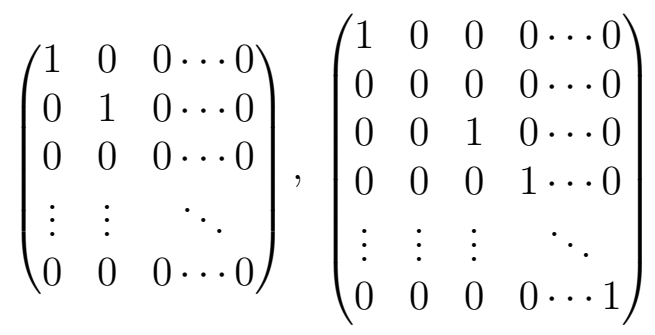

and

$$
\left(\begin{array}{cccc}
0 & 0 & 0 & 0 \cdots 0 \\
0 & 1 & 0 & 0 \cdots 0 \\
0 & 0 & 1 & 0 \cdots 0 \\
0 & 0 & 0 & 1 \cdots 0 \\
\vdots & \vdots & \vdots & \ddots \\
0 & 0 & 0 & 0
\end{array}\right)
$$

\section{When IS ZA $(R)$ Connected?}

A ring $R$ is called semiprimitive if $\operatorname{Jac}(R)=0$, [7]. A ring $R$ is semiprimitive if and only if it is a subdirect product of fields, [8, p. 179].

Theorem 3.1. Let $R$ be a semiprimitive ring. If at least one of the maximal ideals of $R$ is principal, then $\mathrm{ZA}(R)$ is a connected graph with $\operatorname{diam}(\mathrm{ZA}(R)) \leq 4$.

Proof. Suppose that $\mathfrak{m}$ is a maximal ideal of $R$ where $\mathfrak{m}=R t$ for some $t \in R$. Let $x, y$ be two different nonzero nonunit elements of $R$. Consider the following cases.

Case 1. Let $x, y \notin \mathfrak{m}$. Then $R x+\mathfrak{m}=R$ and $R y+\mathfrak{m}=R$. Hence $x, y$ are adjacent to $t$. So $d_{\mathrm{ZA}(R)}(x, y) \leq 2$.

Case 2. Let $x \in \mathfrak{m}$ and $y \notin \mathfrak{m}$. Notice that $y$ is adjacent to $t$. Since $\operatorname{Jac}(R)=\{0\}$, there exists a maximal ideal $\mathfrak{m}^{\prime}$ different from $\mathfrak{m}$ such that $x \notin \mathfrak{m}^{\prime}$. So $R x+\mathfrak{m}^{\prime}=R$, and thus there exist elements $r \in R$ and $z \in \mathfrak{m}^{\prime}$ such that $r x+z=1$. Therefore $\operatorname{Ann}_{R}(x) \cap \operatorname{Ann}_{R}(z)=\{0\}$. So $x$ is adjacent to $z$. Clearly $z \notin \mathfrak{m}$. Then $z$ is adjacent to $t$. Hence $d_{\mathrm{ZA}(R)}(x, y) \leq 3$.

Case 3. Let $x, y \in \mathfrak{m}$. A manner similar to Case 2 shows that $d_{\mathrm{ZA}(R)}(x, t) \leq 2$ and $d_{\mathrm{ZA}(R)}(y, t) \leq 2$. Therefore $d_{\mathrm{ZA}(R)}(x, y) \leq 4$. 
Consequently $\mathrm{ZA}(R)$ is a connected graph with $\operatorname{diam}(\mathrm{ZA}(R)) \leq 4$.

Theorem 3.2. Let $R$ be a Bézout ring. If $\mathrm{ZA}(R)$ is connected, then one of the following conditions holds:

(a) there exists a nonzero nonunit element $x$ of $R$ such that $\operatorname{Ann}_{R}(x)=\{0\}$;

(b) $\operatorname{Jac}(R)=\{0\}$;

(c) $\operatorname{Jac}(R)=\{0, x\}$ where $x$ is the only nonzero nonunit element of $R$.

Proof. Assume that for every nonzero nonunit element $x$ of $R, \operatorname{Ann}_{R}(x) \neq\{0\}$ and also $\operatorname{Jac}(R) \neq\{0\}$. Let $x$ be a nonzero element in $\operatorname{Jac}(R)$. Suppose that ZA $(R)$ has a vertex $y$ different from $x$. Thus $R x+R y=R z$ for some $z \in R$, because $R$ is a Bézout ring. Notice that $y \in \mathfrak{m}$ for some maximal ideal $\mathfrak{m}$ of $R$. Hence $z$ is nonzero nonunit and so by assumption $\operatorname{Ann}_{R}(z) \neq\{0\}$, which shows that $x$ and $y$ are not adjacent. This contradiction implies that $|V(\mathrm{ZA}(R))|=1$, and so $\operatorname{Jac}(R)=\{0, x\}$.

As a direct consequence of Theorem 3.1 and Theorem 3.2 we have the following result.

Corollary 3.1. Let $R$ be a Bézout ring such that at least one of the maximal ideals of $R$ is principal. Then $\mathrm{ZA}(R)$ is connected if and only if one of the following conditions holds:

(a) there exists a nonzero nonunit element $x$ of $R$ such that $\operatorname{Ann}_{R}(x)=\{0\}$;

(b) $\operatorname{Jac}(R)=\{0\}$;

(c) $\operatorname{Jac}(R)=\{0, x\}$ where $x$ is the only nonzero nonunit element of $R$.

Theorem 3.3. Let $R=F_{1} \times F_{2} \times \cdots \times F_{n}$ where $F_{i}$ 's are fields. Then $\mathrm{ZA}(R)$ is a connected graph with

$$
\operatorname{diam}(\mathrm{ZA}(R))= \begin{cases}1, & \text { if } n=2 \text { and }\left|F_{1}\right|=\left|F_{2}\right|=2 \\ 2, & \text { if } n=2 \text { and either }\left|F_{1}\right|>2 \text { or }\left|F_{2}\right|>2, \\ 3, & \text { if } n \geq 3 .\end{cases}
$$

Proof. Let $n=2$. In this case every vertex in $\mathrm{ZA}(R)$ is of the form $(u, 0)$ or $(0, v)$ where $u \neq 0$ and $v \neq 0$. Furthermore, two vertices $(u, 0)$ and $(0, v)$ are adjacent.

In the case when $n=2$ and $\left|F_{1}\right|=\left|F_{2}\right|=2$, we have $R \simeq \mathbb{Z}_{2} \times \mathbb{Z}_{2}$. So $\mathrm{ZA}(R) \simeq K_{2}$.

Let $n=2$ and $\left|F_{1}\right|>2$. In this case, every two different vertices $\left(u_{1}, 0\right)$ and $\left(u_{2}, 0\right)$ cannot be adjacent. On the other hand $\left(u_{1}, 0\right)$ and $\left(u_{2}, 0\right)$ are adjacent to $(0,1)$. So $\mathrm{d}_{\mathrm{ZA}(R)}\left(\left(u_{1}, 0\right),\left(u_{2}, 0\right)\right)=2$. Hence $\operatorname{diam}(\mathrm{ZA}(R))=2$.

Now, let $n \geq 3$. Assume that $u=\left(u_{1}, u_{2}, \ldots, u_{n}\right)$ and $v=\left(v_{1}, v_{2}, \ldots, v_{n}\right)$ are two different vertices. There exist two indexes $i, j$ such that $u_{i} \neq 0$ and $v_{j} \neq 0$. So $u=\left(u_{1}, u_{2}, \ldots, u_{n}\right)$ is adjacent to $(1, \ldots, 1, \overbrace{0}^{i-\text { th }}, 1, \ldots, 1)$. Also $v=\left(v_{1}, v_{2}, \ldots, v_{n}\right)$ is adjacent to $(1, \ldots, 1, \overbrace{0}^{j-\text { th }}, 1, \ldots, 1)$. If $i \neq j$, then the vertex $(1, \ldots, 1, \overbrace{0}^{i-\text { th }}, 1, \ldots, 1)$ 
is adjacent to $(1, \ldots, 1, \overbrace{0}^{j-\text { th }}, 1, \ldots, 1)$. Thus $\mathrm{ZA}(R)$ is connected and $d_{\mathrm{ZA}(R)}(u, v) \leq 3$. In special case, we have the following path

$$
(0,1,0, \ldots, 0)-(1,0,1, \ldots, 1)-(0,1, \ldots, 1)-(1,0, \ldots, 0) .
$$

Consequently $\operatorname{diam}(\mathrm{ZA}(R))=3$.

\section{When IS ZA $(R)$ Star?}

Lemma 4.1. Let $R$ be a ring. If $\mathrm{ZA}(R)$ is a star, then $|\operatorname{Max}(R)| \leq 2$.

Proof. Suppose that $\mathrm{ZA}(R)$ is a star. If $\mathfrak{m}$ and $\mathfrak{m}^{\prime}$ are two different maximal ideals of $R$, then for every $x \in \mathfrak{m} \backslash \mathfrak{m}^{\prime}$ we have $R x+\mathfrak{m}^{\prime}=R$. Hence there exist elements $r \in R$ and $y \in \mathfrak{m}^{\prime} \backslash \mathfrak{m}$ such that $r s+y=1$. Therefore $\operatorname{Ann}_{R}(x) \cap \operatorname{Ann}_{R}(y)=\{0\}$. So $x$ and $y$ are adjacent. Let $\mathfrak{m}_{1}, \mathfrak{m}_{2}$ and $\mathfrak{m}_{3}$ be three different maximal ideals of $R$. Then there are elements $a \in \mathfrak{m}_{1} \backslash\left(\mathfrak{m}_{2} \cup \mathfrak{m}_{3}\right), b \in \mathfrak{m}_{2} \backslash\left(\mathfrak{m}_{1} \cup \mathfrak{m}_{3}\right)$ and $c \in \mathfrak{m}_{3} \backslash\left(\mathfrak{m}_{1} \cup \mathfrak{m}_{2}\right)$. Then either $a, b, c$ are pairwise adjacent or there exist at least two disjoint edges in $\mathrm{ZA}(R)$, which is a contradiction. Consequently $|\operatorname{Max}(R)| \leq 2$.

Theorem 4.1. Let $R$ be a Bézout ring that is not a field. Then $\mathrm{ZA}(R)$ is a star if and only if one of the following conditions holds:

(a) $(R, \mathfrak{m})$ when $\mathfrak{m}=\{0, x\}$ in which $x$ is a nonzero element of $R$ with $x^{2}=0$;

(b) $R \simeq \mathbb{Z}_{2} \times F$ where $F$ is a field.

Proof. $(\Rightarrow)$ Suppose that $\mathrm{ZA}(R)$ is a star. Hence $|\operatorname{Max}(R)| \leq 2$, by Lemma 4.1. Notice that if $\operatorname{Ann}_{R}(t)=\{0\}$ for some element $t$ of a maximal ideal $\mathfrak{m}$, then $\left\{t^{n} \mid n \in \mathbb{N}\right\}$ is an infinite clique that is impossible. Consider the following cases:

Case 1. $\operatorname{Max}(R)=\{\mathfrak{m}\}$. Let $x$ be a nonzero element in $\mathfrak{m}$. Then by Theorem 2.1, $\mathrm{ZA}(R)$ is empty and so $\mathfrak{m}=\{0, x\}$. On the other hand, by Nakayama's Lemma we have that $x^{2}=0$.

Case 2. $\operatorname{Max}(R)=\left\{\mathfrak{m}_{1}, \mathfrak{m}_{2}\right\}$. Since $\mathfrak{m}_{1}+\mathfrak{m}_{2}=R$, there exist $x \in \mathfrak{m}_{1}$ and $y \in \mathfrak{m}_{2}$ such that $x+y=1$. Hence $x$ and $y$ are adjacent. Now, if there exists $0 \neq z \in \mathfrak{m}_{1} \cap \mathfrak{m}_{2}$, then $z$ is not adjacent to $x$ and $y$, because $R$ is a Bézout ring and $\operatorname{Ann}_{R}(t)=\{0\}$ for every nonzero nonunit element $t$ of $R$. This contradiction shows that $\mathfrak{m}_{1} \cap \mathfrak{m}_{2}=\{0\}$. Hence by Chinese Remainder Theorem we deduce that $R \simeq R / \mathfrak{m}_{1} \oplus R / \mathfrak{m}_{2}$. If there exist nozero elements $a_{1}, a_{2} \in R / \mathfrak{m}_{1}$ and $b_{1}, b_{2} \in R / \mathfrak{m}_{2}$, then we have the following path

$$
\left(a_{1}, 0\right)-\left(0, b_{1}\right)-\left(a_{2}, 0\right)-\left(0, b_{2}\right),
$$

a contradiction. Hence we can assume that $R / \mathfrak{m}_{1}=\mathbb{Z}_{2}$.

$(\Leftarrow)$ If $($ a) holds, then clearly ZA $(R)$ is a star. Assume that (b) holds. Notice that $(1,0)$ is adjacent to all vertices $(0, u)$ where $u$ is a nonzero element of $F$. Also, for every two different elements $u_{1}, u_{2} \in F,\left(0, u_{1}\right)$ and $\left(0, u_{2}\right)$ are not adjacent. Consequently $\mathrm{ZA}(R)$ is a star. 


\section{When is ZA $(R)$ Complete?}

Proposition 5.1. Let $R$ be a ring. If $\mathrm{ZA}(R)$ is a complete graph, then $\mathcal{A}_{R}$ is a complete graph.

Proof. Assume that $\mathrm{ZA}(R)$ is a complete graph. Let $I, J$ be two nonzero proper ideals of $R$. Then there are two different nonzero nonunit elements $x, y \in R$ such that $x \in I$ and $y \in J$. Hence $\operatorname{Ann}_{R}(I) \cap \operatorname{Ann}_{R}(J) \subseteq \operatorname{Ann}_{R}(x) \cap \operatorname{Ann}_{R}(y)=\{0\}$. Therefore $I$ and $J$ are adjacent.

The following remark shows that the converse of Proposition 5.1 is not true.

Remark 5.1. Consider the ring $R=\mathbb{Z}_{5} \times \mathbb{Z}_{5}$. By [1, Theorem 6], $\mathcal{A}_{R}\left(=K_{2}\right)$ is a complete graph. But $\mathrm{ZA}(R)$ is a 4 -regular graph that is not a complete graph.

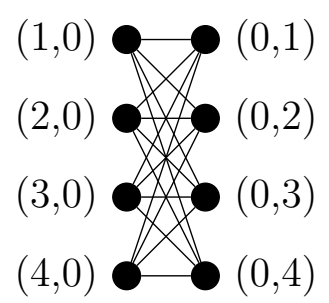

FIGURE 1. ZA $(R)$

Theorem 5.1. Let $R$ be a ring. Then $\mathrm{ZA}(R)$ is a complete graph if and only if one of the following conditions holds:

(a) $R$ has exactly one nonzero nonunit element;

(b) $R$ is an integral domain;

(c) $R=\mathbb{Z}_{2} \times \mathbb{Z}_{2}$.

Proof. $(\Rightarrow)$ Assume that $\mathrm{ZA}(R)$ is a complete graph. Then, by Proposition $5.1, \mathcal{A}_{R}$ is a complete graph. Suppose that $R$ is not an integral domain. So there exists a nonzero nonunit element $x \in R$ such that $\operatorname{Ann}_{R}(x) \neq\{0\}$. Therefore, [1, Theorem 6] implies that either $R$ has exactly one nonzero proper ideal or $R$ is a direct product of two fields. Suppose that the former case holds. If $y$ is a nonzero nonunit element of $R$ different from $x$, then $R x=R y$. So $A_{R}(x) \cap \operatorname{Ann}_{R}(y)=\operatorname{Ann}_{R}(x) \neq\{0\}$, which is a contradiction. Therefore $R$ has exactly one nonzero nonunit element. Now, let $R$ be a direct product of two fields, say $R=F_{1} \times F_{2}$. If there exist two different nonzero elements $u, v$ in $F_{1}$, then $(u, 0)$ and $(v, 0)$ cannot be adjacent. Hence $F_{1}=\mathbb{Z}_{2}$. Similarly, we can show that $F_{2}=\mathbb{Z}_{2}$. Consequently $R=\mathbb{Z}_{2} \times \mathbb{Z}_{2}$.

$(\Leftarrow)$ Clearly, if $(\mathrm{a})$ or $(\mathrm{b})$ holds, then $\mathrm{ZA}(R)$ is a complete graph. Assume that (c) holds. Then $\mathrm{ZA}(R) \simeq K_{2}$ and we are done.

\section{Chromatic Number and Clique Number of ZA $(R)$}

Recall that, a ring $R$ is said to be reduced if it has no nonzero nilpotent elements. 
Theorem 6.1. If $R$ is a reduced Noetherian ring, then the chromatic number of $\mathrm{ZA}(R)$ is infinite or $R$ is a direct product of finitely many fields.

Proof. The proof is similar to that of [1, Theorem 16].

Lemma 6.1. Let $P_{1}$ and $P_{2}$ be two prime ideals of a ring $R$ with $P_{1} \cap P_{2}=\{0\}$. Then every two nonzero elements $x \in P_{1}$ and $y \in P_{2}$ are adjacent.

Proof. Suppose that $r \in \operatorname{Ann}_{R}(x) \cap \operatorname{Ann}_{R}(y)$. Since $r x=0 \in P_{2}$ and $x \notin P_{2}$, then $r \in P_{2}$. Similarly it turns out that $r \in P_{1}$. Hence $r \in P_{1} \cap P_{2}=\{0\}$.

Theorem 6.2. Let $R$ be a ring and $n \geq 2$ be a natural number. If either $|\operatorname{Min}(R)|=n$ or $R=R_{1} \times R_{2} \times \cdots \times R_{n}$ where $R_{i}$ 's are rings, then $\omega(\mathrm{ZA}(R)) \geq n$.

Proof. Assume that $\operatorname{Min}(R)=\left\{\mathfrak{p}_{1}, \mathfrak{p}_{2}, \ldots, \mathfrak{p}_{n}\right\}$ where $\mathfrak{p}_{i}$ 's are nonzero. So, by Lemma $6.1, n \leq \omega(\mathrm{ZA}(R))$. Now, suppose that $R=R_{1} \times R_{2} \times \cdots \times R_{n}$ where $R_{i}$ 's are rings. Then $\{(1, \ldots, 1, \overbrace{0}^{i-\text { th }}, 1, \ldots, 1) \mid 1 \leq i \leq n\}$ is a clique in $\mathrm{ZA}(R)$ and the result follows.

\section{When IS ZA $(R) k$-REGUlaR?}

Recall that a finite field of order $q$ exists if and only if the order $q$ is a prime power $p^{s}$. A finite field of order $p^{s}$ is denoted by $\mathbb{F}_{p^{s}}$.

Theorem 7.1. Let $R$ be a Bézout ring with $|\operatorname{Max}(R)|<\infty$. Then $\mathrm{ZA}(R)$ is a k-regular graph $(0<k<\infty)$ if and only if $R \simeq \mathbb{F}_{k+1} \times \mathbb{F}_{k+1}$.

Proof. The "if" part has a routine verification. Let ZA $(R)$ be a $k$-regular graph $(0<$ $k<\infty)$. If $\operatorname{Ann}_{R}(x)=\{0\}$ for some nonzero nonunit element $x$ of $R$, then $\left\{x^{n} \mid n \in\right.$ $\mathbb{N}\}$ is an infinite clique that is a contradiction. Then, for every nonzero nonunit element $x$ of $R$ we have $\operatorname{Ann}_{R}(x) \neq\{0\}$. Similar to the manner that described in the proof of Theorem 2.3, we have $R \simeq F_{1} \times F_{2} \times \cdots \times F_{n}$ where $F_{i}$ 's are fields and $n=|\operatorname{Max}(R)|$. Since $\operatorname{Ann}_{R}((1,0, \ldots, 0))=0 \times F_{2} \times F_{3} \times \cdots \times F_{n}$ and $\operatorname{Ann}_{R}((0,1,0, \ldots, 0))=$ $F_{1} \times 0 \times F_{3} \times \cdots \times F_{n}$, then

$$
\mathrm{N}_{\mathrm{ZA}(R)}((1,0, \ldots, 0))=\left\{\left(0, u_{2}, \ldots, u_{n}\right) \mid u_{i} \in F_{i} \backslash\{0\} \text { for } 2 \leq i \leq n\right\}
$$

and

$$
\mathrm{N}_{\mathrm{ZA}(R)}((0,1,0, \ldots, 0))=\left\{\left(u_{1}, 0, u_{3}, \ldots, u_{n}\right) \mid u_{i} \in F_{i} \backslash\{0\} \text { for } 1 \leq i \leq n, i \neq 2\right\} .
$$

So

$$
\left(\left|F_{2}\right|-1\right)\left(\left|F_{3}\right|-1\right) \cdots\left(\left|F_{n}\right|-1\right)=\left(\left|F_{1}\right|-1\right)\left(\left|F_{3}\right|-1\right) \cdots\left(\left|F_{n}\right|-1\right)
$$

because $\mathrm{ZA}(R)$ is $k$-regular. Hence $\left|F_{1}\right|=\left|F_{2}\right|$. Similarly we can show that $\left|F_{1}\right|=$ $\left|F_{2}\right|=\cdots=\left|F_{n}\right|$. Let $n \geq 3$. Note that $\mathrm{N}_{\mathrm{ZA}(R)}((1,1,0, \ldots, 0))$ is the union of the following sets

$$
\left\{\left(u_{1}, 0, u_{3}, \ldots, u_{n}\right) \mid u_{i} \in F_{i} \backslash\{0\} \text { for } 1 \leq i \leq n, i \neq 2\right\},
$$




$$
\left\{\left(0, u_{2}, \ldots, u_{n}\right) \mid u_{i} \in F_{i} \backslash\{0\} \text { for } 2 \leq i \leq n\right\}
$$

and

Therefore,

$$
\left\{\left(0,0, u_{3}, \ldots, u_{n}\right) \mid u_{i} \in F_{i} \backslash\{0\} \text { for } 3 \leq i \leq n\right\}
$$

$$
\left(\left|F_{1}\right|-1\right)^{n-1}=2\left(\left|F_{1}\right|-1\right)^{n-1}+\left(\left|F_{1}\right|-1\right)^{n-2},
$$

since $\mathrm{ZA}(R)$ is $k$-regular. Thus $\left|F_{1}\right|=0$ which is a contradiction. Consequently $n=2$. If there exist two different nonzero elements $u, u^{\prime}$ in $F_{1}$, then $(u, 0)$ and $\left(u^{\prime}, 0\right)$ cannot be adjacent. On the other hand for every nonzero elements $u \in F_{1}$ and $v \in F_{2},(u, 0)$ and $(0, v)$ are adjacent. So $\operatorname{deg}_{\mathrm{ZA}(R)}((u, 0))=\left|F_{1}\right|-1=k$. Therefore $R \simeq \mathbb{F}_{k+1} \times \mathbb{F}_{k+1}$.

Corollary 7.1. Let $R$ be a Bézout ring with $|\operatorname{Max}(R)|<\infty$. If $\mathrm{ZA}(R)$ is a k-regular graph $(0<k<\infty)$, then $k+1$ is a prime power.

Acknowledgements. The author is deeply grateful to the referee for careful reading of the original manuscript and the valuable suggestions.

\section{REFERENCES}

[1] S. Akbari, A. Alilou, J. Amjadi and S. M. Sheikholeslami, The co-annihilating ideal graphs of commutative rings, Canad. Math. Bull. 60 (2017), 3-11.

[2] D. F. Anderson and D. Dobbs, Zero-dimensional Commutative Rings, Lecture Notes in Pure and Applied Mathematics, Marcel Dekker, Inc. New York, Basel, Hong Kong, 1995.

[3] D. F. Anderson and P. S. Livingston, The zero-divisor graph of a commutative ring, J. Algebra, 217 (1999), 434-447.

[4] D. D. Anderson and M. Naseer, Beck's coloring of a commutative ring, J. Algebra, 159 (1993), $500-514$.

[5] A. Badawi, On the annihilator graph of a commutative ring, Comm. Algebra, 42 (2014), 108-121.

[6] I. Beck, Coloring of commutative rings, J. Algebra, 116 (1988), 208-226.

[7] T. Y. Lam, A First Course in Noncommutative Rings, Springer-Verlag New York, Inc. 1991.

[8] T. Y. Lam, Exercises in Classical Ring Theory, Springer-Verlag New York, Inc. 2003.

[9] S. P. Redmond, An ideal-based zero-divisor graph of a commutative ring, Comm. Algebra, 31 (2003), 4425-4443.

${ }^{1}$ Department of Mathematics and Applications, University of MoHaghegh ARdabili,

P.O.Pox. 179, Ardabil, Iran

Email address: h.mostafanasab@gmail.com 